\title{
Image Quality Assessment for Extended-Volume C-arm CT Using a Multi-Turn Reverse Helix
}

\author{
Z. Yu, A. Maier, G. Lauritsch, F. Vogt, M. Schönborn, C. Köhler, J. Hornegger and F. Noo
}

\begin{abstract}
C-arm computed tomography (CT) with axially extended field-of-view is valuable when imaging a long organ is desired in the interventional room. However, current C-arm CT employs a circular short scan that only provides incomplete data and short axial coverage. To enable long-object 3D imaging capability on a $\mathrm{C}$-arm system, a multi-turn reverse helix is an attractive solution for data acquisition. We have implemented this trajectory on a state-of-the-art multi-axis C-arm system and performed image reconstruction using our Fusion-RFDK method. This work evaluates these reconstruction results by comparing them with those obtained from a circular short scan. We observed comparable image quality between the two source trajectories.
\end{abstract}

\section{INTRODUCTION}

$\mathrm{C}$-arm computed tomography (CT) is an innovative imaging technique that enables a $\mathrm{C}$-arm system to generate $3 \mathrm{D}$ images like a CT system. Clinical reports [1] show that this technique is valuable in the interventional room. However, current $\mathrm{C}$-arm CT employs a circular short scan for data acquisition that only produces incomplete projection data and limits axial coverage in the direction of the patient table. This short coverage was reported as a problem in hepatic vascular interventions [1]. In general, this limitation is an issue whenever imaging a long organ is desired in intra-operative cases, e.g., in treatment of aortic diseases.

Recently, we have developed a framework for C-arm CT with axially extended field-of-view (FOV) [2]. In this framework, a reverse helical source trajectory [3] was implemented on a state-of-the-art C-arm system for cone-beam data acquisition, and our Fusion-RFDK algorithm [4] was employed for image reconstruction. This work performs an image quality assessment for the reconstruction results obtained from such a framework. These reconstructions are compared to state-ofthe-art C-arm CT images, i.e., images obtained from a single circular short scan.

\section{Materials And Methods}

We implemented a five-turn reverse helix on a state-of-theart multi-axis C-arm system (Artis zeego, Siemens AG, Forchheim, Germany). The reverse helix is depicted in Figure 1, whereas the parameters of the $\mathrm{C}$-arm system are listed in

Zhicong Yu was formerly with the Department of Radiology, the University of Utah, Salt Lake City, UT, USA; he is currently with the Department of Radiology, Mayo Clinic, Rochester, MN, USA (e-mail: zhicongyu@gmail.com). Frederic Noo is with the Department of Radiology, the University of Utah, Salt Lake City, USA (e-mail: noo@ucair.med.utah.edu). Andreas Maier, Joachim Hornegger are with the Pattern Recognition Lab, FriedrichAlexander-University of Erlangen-Nuremberg, Erlangen, Bavaria, Germany. Günter Lauritsch, Florian Vogt, Manfred Schönborn and Christoph Köhler are with the Siemens AG Healthcare, Forchheim, Bavaria, Germany.
Table I. Each helical turn consisted of 681 projections with an angular step-size $0.35^{\circ}$ and an axial translation step-size $0.088 \mathrm{~mm}$. For comparison, we also implemented a circular short scan on the same $\mathrm{C}$-arm system, which consisted of 638 projections with an angular step-size $0.37^{\circ}$.

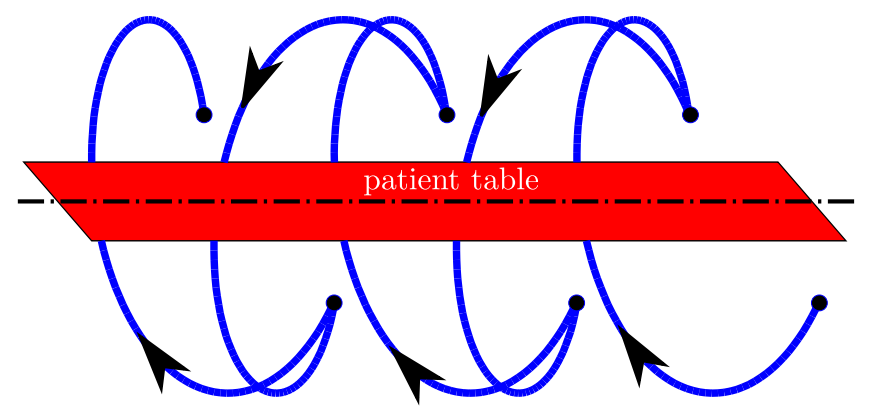

Fig. 1. Depiction of the five-turn reverse helix.

TABLE I

PARAMETERS OF THE STATE-OF-THE-ART MULTI-AXIS C-ARM SYSTEM

\begin{tabular}{ll}
\hline \hline Parameter & Value \\
\hline Source-to-detector distance & $1200 \mathrm{~mm}$ \\
Detector A/D converter & $16 \mathrm{bits}$ \\
Detector size & $300 \mathrm{~mm} \times 400 \mathrm{~mm}$ \\
Detector position mode & landscape \\
Detector pixel size & $154 \mu \mathrm{m} \times 154 \mu \mathrm{m}$ \\
Detector binning mode & $2 \times 2$ \\
Source-to-rotational-axis distance & $785 \mathrm{~mm}$ \\
\hline \hline
\end{tabular}

We selected two phantoms for data acquisition. The first one was a torso phantom embedded with a SAWBONES spine (Sawbones Europe AB, Malmö, Sweden). This phantom was placed in the prone position and the entire SAWBONES spine was within the field-of-view(FOV); see the left of Figure 2. The second one was a combination of the CATPHAN phantom (The Phantom Laboratory, Greenwich, USA) and the Siemens CB phantom(QRM, Möhrendorf, Germany), which were placed in-line along their axial direction to emulate a long object; see the right of Figure 2. We call the combined phantom the CATSCB phantom.

Projection data of the above two phantoms were acquired as follows. For the reverse helix, the torso phantom and the CATSCB phantom were scanned using (90 kVp, $43 \mathrm{~mA})$ and $(91.2 \mathrm{kVp}, 122.8 \mathrm{~mA})$, respectively. The corresponding dose-area-product(DAP) measurements are listed in Table II. For the circular trajectory, the torso phantom and the CATSCB phantom were scanned using (90 kVp, $43 \mathrm{~mA}$ ) and $(90 \mathrm{kVp}, 137 \mathrm{~mA})$ respectively, which corresponded to DAP 

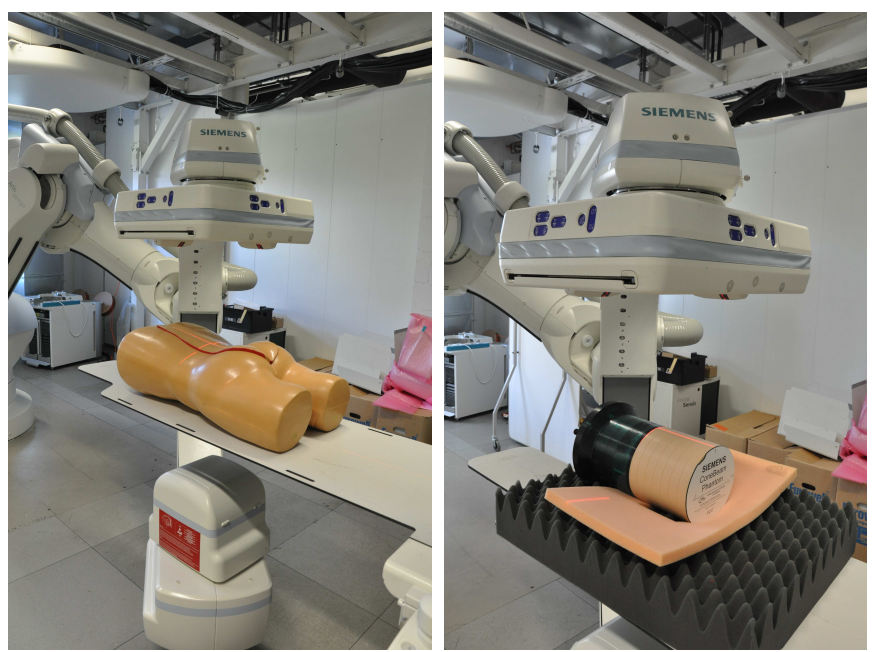

Fig. 2. Left: the torso phantom embedded with a SAWBONES spine. Right: the CATSCB phantom composed by the CATPHAN phantom and the Siemens CB phantom.

measurements of $60.45 \mathrm{mGy} \cdot \mathrm{cm}^{2}$ and $304.96 \mathrm{mGy} \cdot \mathrm{cm}^{2}$ respectively. Note that the numbers for the circular scans were comparable to but not exactly the same as those for the reverse helical scans. These slight differences were due to utilization of pre-defined scanning protocols.

TABLE II

DOSE-AREA-PRODUCT MEASUREMENTS FOR THE FIVE-TURN REVERSE HELIX $\left(\mathrm{MGY} \cdot \mathrm{CM}^{2}\right)$

\begin{tabular}{l|lllll|l}
\hline \hline & $1 \mathrm{st}$ & 2nd & 3rd & 4th & 5th & total \\
\hline Torso & 60.27 & 60.60 & 60.59 & 60.61 & 60.64 & 302.70 \\
\hline CATSCB & 314.60 & 315.54 & 315.71 & 315.65 & 315.41 & 1576.91 \\
\hline \hline
\end{tabular}

The projection data were next preprocessed using the overexposure, scatter and beam hardening correction methods that were similar to those presented in [5]. Note that these data correction methods were optimized for the circular trajectory, but not for the reverse helix. Furthermore, both the reverse helix and the circular trajectory were calibrated based on the method in [6]. Finally, the reverse helix images were reconstructed using our Fusion-RFDK (ramp kernel based FDK) method [4], whereas the circular images were reconstructed using the RFDK method. The 3D images from the reverse helix and the circular trajectory were of axial length $274 \mathrm{~mm}$ and $160 \mathrm{~mm}$, respectively.

\section{Image Quality Assessment}

We first performed image quality comparison between the two trajectories by visual inspections. Slightly low-frequency differences were observed between the results from the reverse helix and those from the circular trajectory. These differences were seen as a combination of CB artifacts and residual errors from physical effects. To account for these low-frequency differences in image display, a display anchor called $\mu_{\text {anchor }}$ was introduced. The values of the anchors were calculated using small uniform regions indicated either by squares or circles in each image.
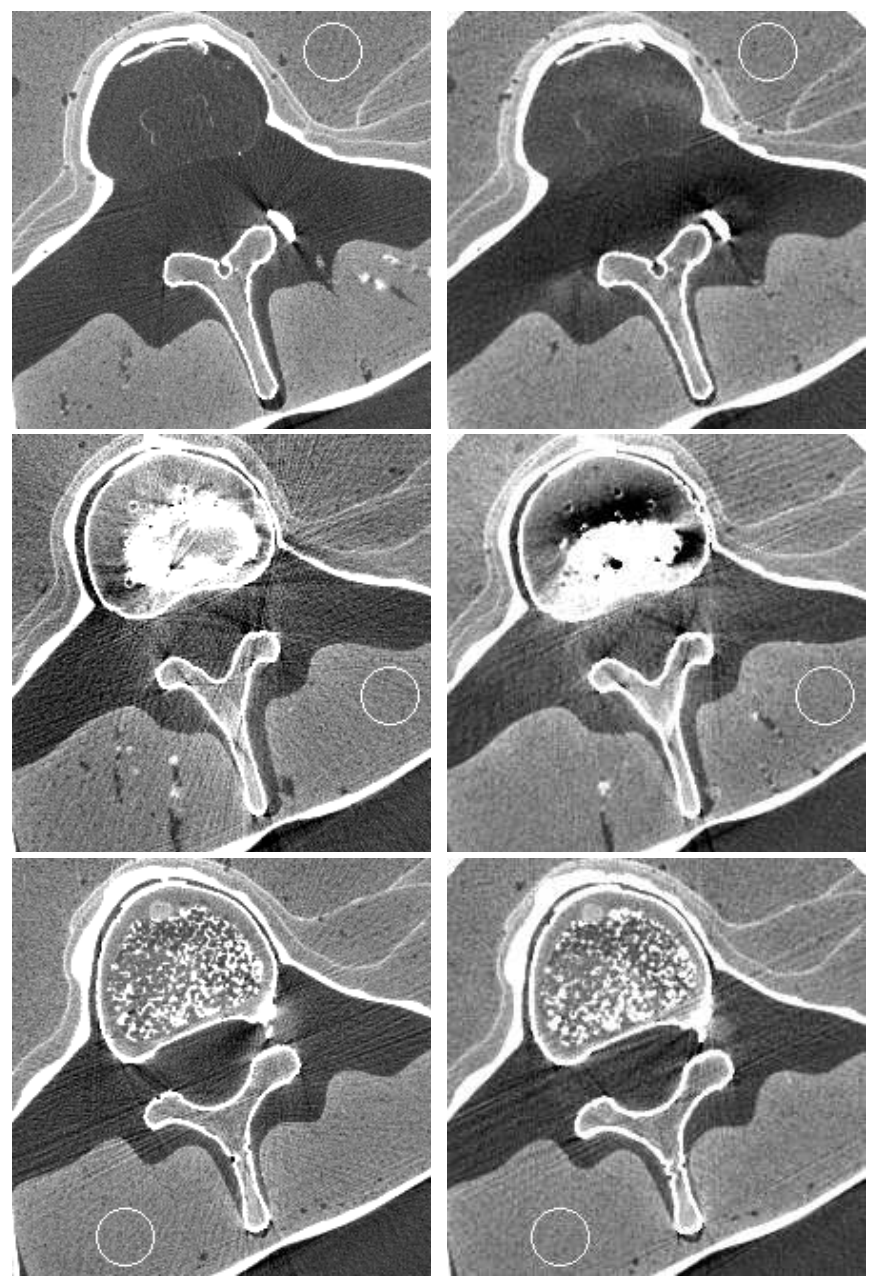

Fig. 3. Transverse views of the reconstructions of the torso phantom. Left column: results from the reverse helical trajectory. Right column: results from the circular trajectory. Display window: $\mu_{\text {anchor }}+[-500,500] \mathrm{HU}$; voxel sidelength: $0.5 \mathrm{~mm}$.

Images appeared differently in the transverse direction from in the sagittal direction. In the transverse direction, images from the two trajectories were in general comparable; see Figure 3. In the sagittal direction, $\mathrm{CB}$ artifacts were more uniform and less noticeable for the reverse helix, whereas they significantly varied with slice positions for the circular trajectory; see Figure 4. These observations are consistent with the fact that the reverse helix provides more complete and axially-uniform projection data than the circular trajectory.

We next compared image quality quantitatively using the CATSCB phantom. First, we studied CT number uniformity using the plain section of the Siemens CB phantom; see Figure 5. We observed more capping artifacts for the reverse helix than for the circular trajectory, possibly because the data correction methods used in our experiment were optimized for circular CB images, not for reverse helix images.

Second, we also investigated contrast-to-noise ratio (CNR) using the contrast pattern of the Siemens CB phantom; see Figure 6. We selected four clusters for CNR measurement, namely $\mathcal{G}_{1}, \mathcal{G}_{2}, \mathcal{G}_{3}$, and $\mathcal{G}_{4}$ as shown in Figure 7 . For the reverse helix, the measured CNRs of $\mathcal{G}_{1}, \mathcal{G}_{2}, \mathcal{G}_{3}$, and $\mathcal{G}_{4}$ 

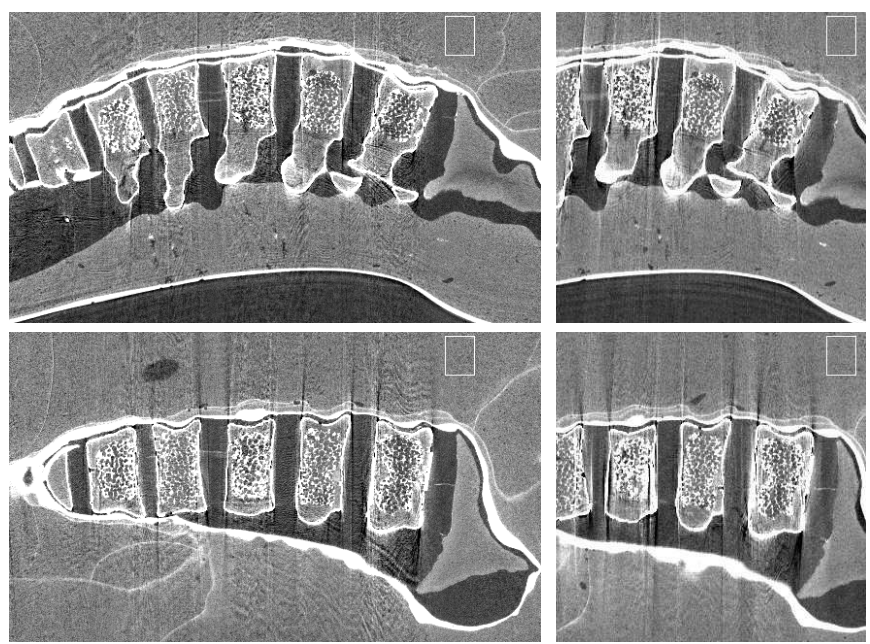

Fig. 4. Axial views of the reconstruction results of the torso phantom. Left: $274 \mathrm{~mm}$ images from the reverse helix; right: $160 \mathrm{~mm}$ images from the circular trajectory. Top: sagittal view; bottom: coronal view. Display window: $\mu_{\text {anchor }}+[-500,500] \mathrm{HU}$; voxel side-length: $0.5 \mathrm{~mm}$.
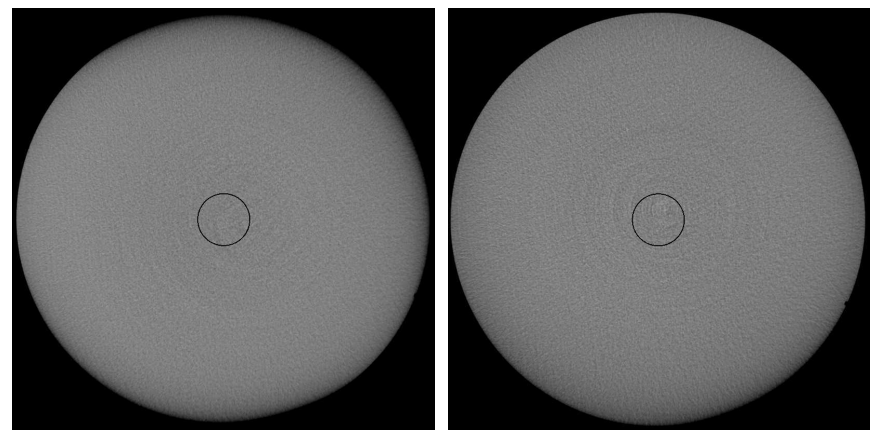

Fig. 5. Comparison of $\mathrm{CT}$ number uniformity using the plain section of the Siemens CB phantom. Left: reverse helix image; right: circular image. Display window: $\mu_{\text {anchor }}+[-500,500] \mathrm{HU}$; voxel side-length: $0.2 \mathrm{~mm}$.
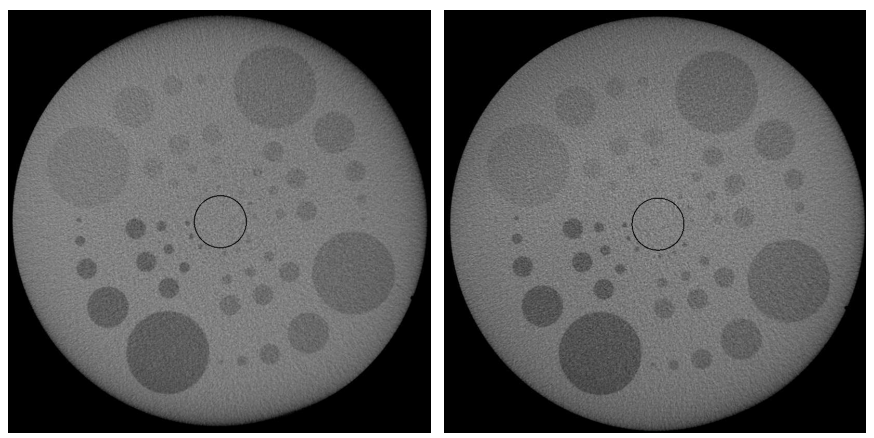

Fig. 6. Comparison of CNR using the contrast section of the Siemens CB phantom. Left: reverse helix image; right: circular image. Display window: $\mu_{\text {anchor }}+[-500,500] \mathrm{HU}$; voxel side-length: $0.2 \mathrm{~mm}$.

were $0.93,1.52,2.00$, and 2.87 , respectively; whereas for the circular trajectory, they were $0.83,1.36,1.81$, and 2.67 , respectively. Note that, the reverse helix image used for CNR calculation was obtained solely using projection data from one helical turn of the reverse helix. Given that the x-ray exposure of one helical turn of the reverse helix was comparable to that of the circular trajectory, the measured CNRs indicate that the two trajectories produced images of comparable CNR.

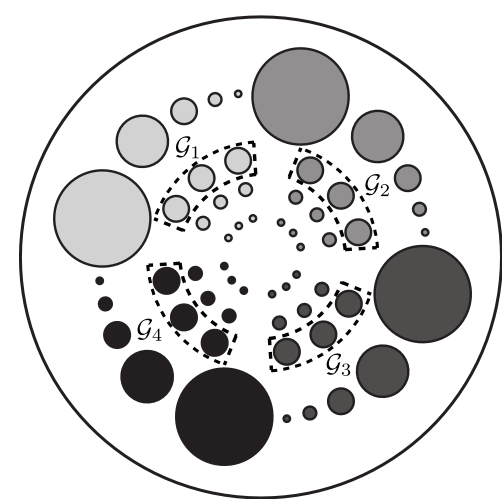

Fig. 7. Illustration of clusters for CNR measurement.
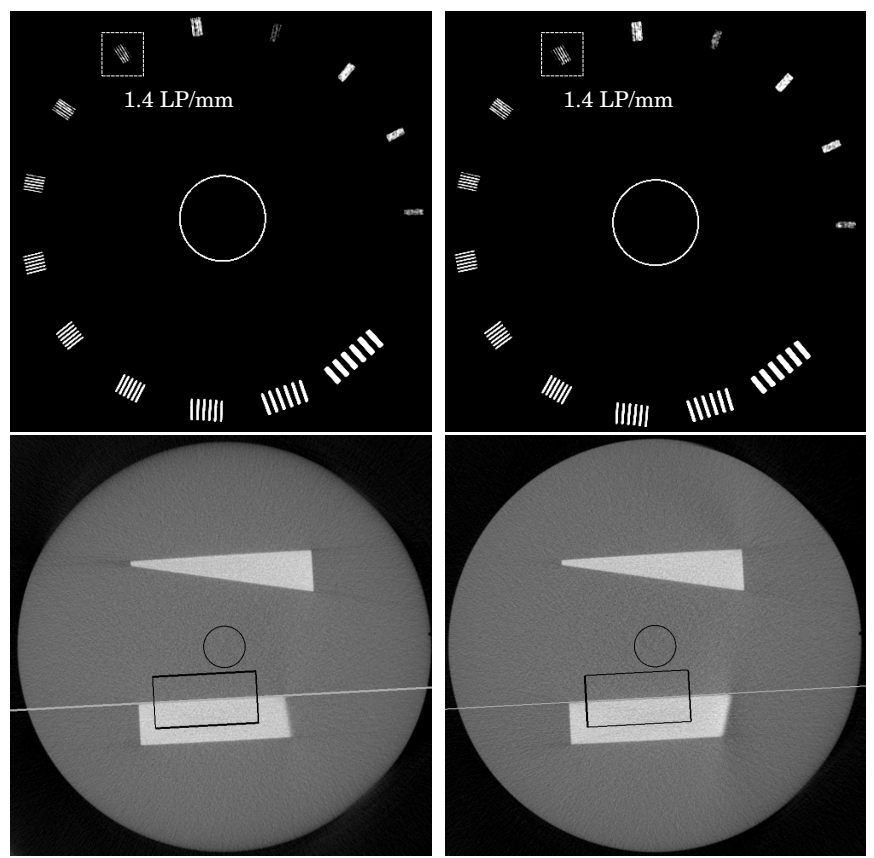

Fig. 8. Comparison of spatial resolution using the Siemens CB phantom. Left: reverse helix images; right: circular images. Top: the resolution section; bottom: the MTF edge section. Display window: $\mu_{\text {anchor }}+[-500,500] \mathrm{HU}$; voxel side-length: $0.2 \mathrm{~mm}$.

Finally, spatial resolution was examined using both the resolution section and the MTF edge section of the Siemens CB phantom; see Figure 8. From visual inspection, reconstruction results of the resolution section from both trajectories exhibit comparable spatial resolution around $1.4 \mathrm{LP} / \mathrm{mm}$. To further verify this observation, we calculated the modulation transfer function(MTF) using the edge spread function associated to the edge indicated in the last row of Figure 8. The resulting MTF curves were similar ; see Figure 9. Note that the maximum detectable spatial frequencies (corresponding to the 0.1 value) for both MTF curves were around $1.3 \mathrm{LP} / \mathrm{mm}$, which is in agreement with the aforementioned visual observation. Hence, both visual inspection and quantitative measurements suggest that the spatial resolution for the reverse helix was comparable to that for the circular trajectory. 


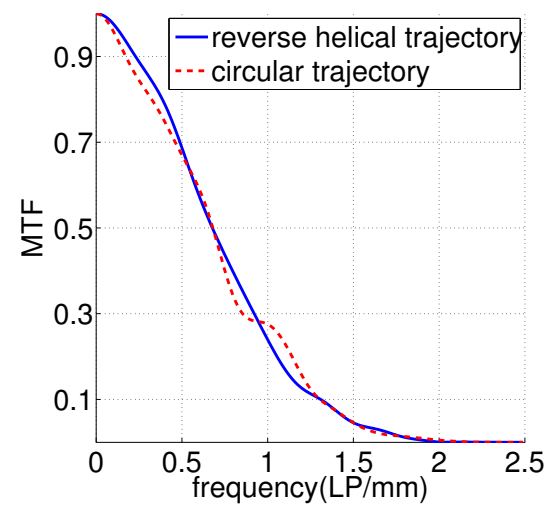

Fig. 9. MTF curves associated to the edges in the bottom row of Fig. 8.

\section{SUMMARY AND OUTLOOK}

We have performed image quality assessment for our previously developed extended-volume $\mathrm{C}$-arm $\mathrm{CT}$ framework, which implemented a five-turn reverse helix on a state-of-theart multi-axis C-arm system. This assessment was achieved through a comparison against a circular short scan using both visual inspection(CB artifacts) and quantitative metrics (capping artifacts, $\mathrm{CNR}$ and MTF). The comparison results demonstrated that satisfactory image quality can be achieved for extended-volume $\mathrm{C}$-arm CT using the reverse helix data acquisition geometry.

Although the framework delivered satisfactory image quality, the reverse helix is not necessarily the optimal source trajectory. Other competitive data acquisition geometries should also be considered for extended-volume C-arm CT, such as arc-extended-line-arc(AELA) [7] and ellipse-lineellipse(ELE) [8]. These two source trajectories are both compatible with the mechanical motion constraints of a $\mathrm{C}$ arm system. Compared to the reverse helix, the AELA and the ELE trajectories are more appropriate for efficient and exact image reconstruction with axially truncated projection data. Currently, we are developing such image reconstruction algorithms for the ELE trajectory. Preliminary results of these algorithms can be found in [9] and [10], and more detailed results will be reported in our future work.

\section{ACKNOWLEDGEMENT}

This work was partially supported by a grant from Siemens Medical Solution USA and by the U.S. National Institutes of Health (NIH) under grants No. R21 EB009168 and R01 EB007236.

\section{DISCLAIMER}

The concepts presented in this paper are based on research and are not commercially available. The product names and/or brands referred to are the property of their respective trademark holders. Its contents are solely the responsibility of the authors and do not necessarily represent the official views of the NIH.

\section{REFERENCES}

[1] M. J. Wallace, M. D. Kuo, C. Glaiberman, C. A. Binkert, R. C. Orth, and G. Soulez, "Three-dimensional C-arm cone-beam CT: applications in the interventional suite," J. Vasc. Interv. Radiol., vol. 19, no. 6, pp. 799-813, Jun. 2008.

[2] Z. Yu, M. Andreas, M. Schönborn, F. Vogt, C. Köhler, G. Lauritsch, J. Hornegger, and F. Noo, "First experimental results on long-object imaging using a reverse helical trajectory with a c-arm system," in Proceedings of The Second International Conference on Image Formation in X-Ray Computed Tomography, F. Noo, Ed., Salt Lake City, USA, Jun. 2012, pp. 364-367.

[3] S. Cho, D. Xia, C. A. Pelizzari, and X. Pan, "Exact reconstruction of volumetric images in reverse helical cone-beam CT," Med. Phys., vol. 35 , no. 7, pp. 3030-3040, Jul. 2008.

[4] Z. Yu, F. Noo, F. Dennerlein, G. Lauritsch, and J. Hornegger, "FDK-type reconstruction algorithms for the reverse helical trajectory," in Nuclear Science Symposium and Medical Imaging Conference Record. Valencia, Spain: IEEE, Oct. 2011, pp. 3980-3985.

[5] M. Zellerhoff, B. Scholz, E.-P. Ruehrnschopf, and T. Brunner, "Low contrast 3D reconstruction from C-arm data," in Proceedings of SPIE, Medical Imaging: Physics of Medical Imaging, M. J. Flynn, Ed., vol. 5745, San Diego, USA, Feb. 2005, pp. 646-655.

[6] N. Strobel, B. Heigl, T. Brunner, O. Schuetz, M. Mitschke, K. Wiesent, and T. Mertelmeier, "Improving 3D image quality of X-ray C-arm imaging systems by using properly designed pose determination systems for calibrating the projection geometry," in Proceedings of SPIE, Meical Imaging: Physics of Medical Imaging, M. J. Yaffe and L. E. Antonuk, Eds., vol. 5030. San Diego, USA: International Society for Optics and Photonics, Feb. 2003, pp. 943-954.

[7] Z. Yu, A. Wunderlich, F. Dennerlein, G. Lauritsch, and F. Noo, "Line plus arc source trajectories and their R-line coverage for long-object cone-beam imaging with a C-arm system," Phys. Med. Biol., vol. 56, no. 12, pp. 3447-3471, May 2011.

[8] Z. Yu, F. Noo, G. Lauritsch, F. Dennerlein, and J. Hornegger, "Ellipseline-ellipse source trajectory and its R-line coverage for long-object cone-beam imaging with a C-arm system," in Proceedings of SPIE, Medical Imaging: Physics of Medical Imaging, N. J. Pelc, R. M. Nishikawa, and B. R. Whiting, Eds., vol. 8313. San Diego, USA: International Society for Optics and Photonics, Feb. 2012, pp. 83 133E183 133E6.

[9] Z. Yu, F. Noo, G. Lauritsch, and J. Hornegger, "Extended-volume image reconstruction using the ellipse-line-ellipse trajectory for a carm system," in Proceedings of the 12th International Meeting on Fully Three-Dimensional Image Reconstruction in Radiology and Nuclear Medicine, Lake Tahoe, USA, 2013, pp. 245-248.

[10] Z. Yu, , G. Lauritsch, J. Hornegger, and F. Noo, "Efficient and exact $\mathrm{C}$-arm cone-beam imaging for axially extended field-of-view using the ellipse-line-ellipse trajectory," in Proceedings of The Third International Conference on Image Formation in X-Ray Computed Tomography, F. Noo, Ed., Salt Lake City, USA, Jun. 2014, pp. 311-314. 